\title{
3 Research Square

\section{Utility of radiomics for predicting patient survival in hepatocellular carcinoma with portal vein tumor thrombosis treated with stereotactic body radiotherapy}

\section{Kui Wu}

The Second Affoliated Hospital, Zhejiang University School of medicine

\section{Yongjie Shui}

The Second Affiliated Hospital, Zhejiang University School of Medicine

\section{Wenzheng Sun}

The Second Affiliated Hospital, Zhejiang University School of Medicine

\section{Sheng Lin}

The Affiliated Hospital of Southwest Medical University

Haowen Pang ( $\sim$ haowenpang@foxmail.com )

The Affiliated Hospital of Southwest Medical University

\section{Research}

Keywords: hepatocellular carcinoma, portal vein tumor thrombosis, stereotactic body radiotherapy, radiomics, outcome prediction

Posted Date: June 4th, 2020

DOI: https://doi.org/10.21203/rs.3.rs-32919/v1

License: (c) (i) This work is licensed under a Creative Commons Attribution 4.0 International License. Read Full License

Version of Record: A version of this preprint was published at Frontiers in Oncology on October 14th, 2020. See the published version at https://doi.org/10.3389/fonc.2020.569435. 


\section{Abstract \\ Objective}

This study aimed to develop and validate the combination of radiomic features and clinical characteristics that can predict patient survival in HCC with PVTT treated with SBRT.

\section{Materials and Methods}

The prediction model was developed in a primary cohort of 70 patients with HCC and PVTT treated with SBRT, using data acquired between December 2015 and June 2017. The radiomic features were extracted from computed tomography (CT) scans. A least absolute shrinkage and selection operator regression model was used to build the radiomic feature. Multivariate Cox-regression hazard models were created for analyzing survival outcomes and the radiomic features and clinical characteristics were presented with a nomogram. The area under the curve (AUC) of the receiver operating characteristic curve was used to evaluate the model. Participants were divided into a high-risk group and a low-risk group based on the radiomic features.

\section{Results}

A total of seven radiomic features and five clinical characteristics were extracted for survival analysis. A combination of the radiomic features and clinical characteristics resulted in better performance for the estimation of overall survival (OS) [AUC $=0.859$ (Cl: $0.770-0.948)]$ than that with clinical characteristics alone $[A U C=0.761(\mathrm{Cl}: 0.641-0.881)]$. These patients were divided into high-risk and low-risk groups according to the radiomic features.

\section{Conclusion}

This study demonstrated that a nomogram of combined radiomic features and clinical characteristics can be conveniently used to facilitate individualized preoperative prediction of OS in patients with HCC with PVTT before SBRT.

\section{Introduction}

Hepatocellular carcinoma (HCC) is the sixth most prevalent cancer worldwide, and the third leading cause of cancer-related death [1]. China accounts for more than $50 \%$ of the global incidence of HCC. HCC is the fourth most commonly diagnosed cancer (2). Macrovascular invasion is common in HCC. Tumor cells invade the portal vein, hepatic veins, or the inferior vena cava in the liver in such cases [3,4]. Portal vein tumor thrombosis (PVTT) is one of the most serious complications of HCC, and is strongly correlated with poor prognosis, with an incidence ranging from 44 to $62.2 \%$ [5]. Between $10 \%$ and $60 \%$ of patients 
with HCC have PVTT at the time of diagnosis [6, 7]. Previous studies have reported that the natural median survival time of patients with HCC and PVTT is 2-4 months $[8,9]$. Several clinical studies have confirmed that radiotherapy is effective for treating HCC with PVTT [10-12].

Stereotactic body radiotherapy (SBRT) has emerged as a new radiotherapy technology that can deliver high doses of radiation to the target area in fewer fractions [13-15]. SBRT can accurately transfer a large dose of multiple beams to the target tumor within 1 to 5 fractions, owing to technical progress in accurate dose transfer, respiratory movement management, and daily image guidance. The relatively short treatment process can benefit patients by reducing interference with other treatment measures. We recommend SBRT patients with unresectable HCC with PVTT for the multidisciplinary treatment of patients with HCC. The purpose of SBRT is to reduce tumor thrombus and retain sufficient portal vein blood flow, to allow the beneficial effect of any follow-up treatment.

Studies have also investigated the possibility of using radiomics as a potential prognostic indicator in oncology, specifically to classify patients and assess their risk categories, to develop personalized oncological treatments [16-18]. The aim of this study was to develop a combination of radiomic features and clinical characteristics to estimate the overall survival (OS) in patients with HCC with PVTT treated using SBRT. Although numerous studies have been published on the use of radiomics in several cancer outcomes prediction models [19-21], and the correlation between the characteristics of radiation and the results of radiotherapy, few studies have focused on HCC with PVTT treated using SBRT. Therefore, our study aimed to develop and validate the combination of radiomic features and clinical characteristics that can predict patient survival in HCC with PVTT treated with SBRT.

\section{Materials And Methods}

\section{Patient selection}

All patients $(n=70)$ who were treated at the Second Affiliated Hospital, Zhejiang University School of Medicine from December 2015 to June 2017 were included in the study. Treatment and data analysis were conducted according to the Declaration of Helsinki. Ethical approval for retrospective data analysis was obtained from the local ethics committee. The diagnosis of liver cancer was based on the guidelines of the American Association for the study of liver diseases [22]. Portal vein invasion can be determined by the presence of filling defects in a low attenuation cavity near the primary tumor, which can be distinguished on enhanced computed tomography (CT).

In this study, patients received SBRT according to the following criteria: (1) tumor thrombus involving the main portal vein and/or the first portal vein, which was deemed unsuitable for surgery or transarterial chemoembolization; (2) Eastern Cooperative Oncology Group (ECOG) performance status (PS) score of $0-1$; (3) absence of refractory ascites; (4) Child-Pugh class A, B, and C; (5) no previous history of radiotherapy for the liver; and (6) availability of more than $700 \mathrm{cc}$ of unaffected liver.

\section{SBRT}


The gross tumor volume (GTV) represents the extent of tumor thrombosis visualized on contrastenhanced CT and magnetic resonance imaging (MRI). If the extent of primary liver disease is small (less than $5 \mathrm{~cm}$ ) and adjacent to the PVTT, both are considered to be a part of the GTV. A total dose of 2550 Gy was prescribed in five fractions over $5-7$ days based on the GTV. SBRT plans were generated using the Varian radiation treatment planning system (Eclipse software, Varian Medical Systems, Palo Alto, CA). Treatment was delivered with a Varian Trilogy linear accelerator (Varian Medical Systems, Palo Alto, $\mathrm{CA}$ ) using a 6-MV photon beam.

Follow-up

The cutoff date for the last follow-up was February 28, 2018 for censored data analysis. The OS was calculated from the start of SBRT to the date of death or the last follow-up visit.

Image acquisition

The entire image used for radiomic analysis was obtained from the CT scan acquired prior to SBRT. Enhanced CT imaging was performed using a LightSpeed RT 16 scanner (GE, USA), with 0.25-cm thick slices, which were analyzed to extract the radiomic features from the GTV that contributed to the SBRT plans. Feature extraction was based on the three-dimensional (3D) slicer platform, and performed using the pyradiomics package, which is available at: http://PyRadiomics.readthedocs.io/en/latest/ (accessed on June 30,2019$)$ [23].

Statistical analyses

All statistical analyses were performed using R software, version 3.6.3 (R Foundation for Statistical Computing, Vienna, Austria) and X-tile software, version 3.6.1 (Yale University School of Medicine, New Haven, Conn). Least absolute shrinkage and selection operator (LASSO) regression modeling was used for data dimension reduction, feature selection, and radiomic feature building. Multivariate Coxregression hazard models were built for the survival outcome, radiomic features, and clinical characteristics presented with the nomogram. The area under the curve (AUC) of the receiver operating characteristic (ROC) curve was used to evaluate the model. The radiomic scores (Rad-scores) were calculated for each patient using a linear combination of selected radiomic features, weighted by their respective coefficients. The cutoff value of the Rad-score was calculated using X-tile software to categorize patients into the high-risk group or low-risk group.

\section{Results}

The median follow-up time was 9.5 months. Twenty-five patients $(35.7 \%)$ were still alive at the time of the current analysis. The median survival time was 10.0 months ( $95 \% \mathrm{Cl}, 7.7-12.3)$. Table 1 shows the patients' clinical characteristics. All 851 radiomic features were extracted, including Shape, First Order statistical, Gray Level Co-occurrence Matrix (GLCM), Gray Level Dependence Matrix (GLDM), Gray Level Run Length Matrix (GLRLM), Gray Level Size Zone Matrix (GLSZM), and Neighboring Gray Tone 
Difference Matrix (NGTDM). High-throughput radiomic features were reduced with LASSO regression (Fig. 1). Four radiomic features and five clinical characteristics were extracted for predict OS analysis. The radiomic features included Short Run Low Gray Level Emphasis of the GLRLM of the wavelet-HLL (feature 1), Idmn of the GLCM of the wavelet-LLL (feature 2), Small Dependence Low Gray Level Emphasis of the GLDM of the wavelet-HLL (feature 3), and Idmn of the GLCM of the original (feature 4). The clinical characteristics included the ECOG score, type of PVTT, Child-Pugh classification, age, albumin and hemoglobin levels. Table 2 summarizes the results of the univariate log-rank test for clinical characteristics. 
Table 1

Patient characteristics

\section{Characteristics}

Age, $y$

$\geq 50$

$<50$

Gender

Male

Female

Stage T

T3

$\mathrm{T} 4$

Stage N

NO

N1

Stage M

M0

M1

Types of PVTT

II

III

IV

HBsAg

Negative

Positive

Child-Pugh classification

A

Abbreviations: PVTT Portal vein tumor thrombus, HBsAg Hepatitis B surface antigen, PS Performance status, ECOG Eastern Cooperative Oncology Group, AFP Alpha-fetoprotein, PLT Platelet, HGB Hemoglobin, TBIL Total bilirubin, ALB Albumin, ALT Alanine aminotransferase, AST Aspartate aminotransferase
$48(68.6)$

$22(31.4)$

$59(84.3)$

$11(15.7)$

65(92.9)

$5(7.1)$

48(68.6)

22(31.4)

57(81.4)

13(18.6)

42 (60.0)

27 (38.6)

1 (1.4)

12 (17.1)

58 (82.9)

45 (64.3) 


\section{Characteristics}

n (\%)

B

24 (34.3)

C

1 (1.4)

ECOG

0

$56(80.0)$

1

14 (20.0)

AFP, ng/L

$\leq 20$

$21 \sim 399$

17 (24.3)

$\geq 400$

40 (57.1)

PLT, $10^{9} / \mathrm{L}$

$\geq 100$

$<100$

31 (44.3)

HGB, g/L

$\geq 120$

$42(60.0)$

$<120$

28 (40.0)

TBIL, $\mu \mathrm{mol} / \mathrm{L}$

$\geq 20$

34 (48.6)

$<20$

36 (51.4)

ALB, g/L

$\geq 35$

41 (58.6)

$<35$

29 (41.4)

ALT, U/L

$\geq 50$

25 (35.7)

$<50$

45 (64.3)

AST, U/L

Abbreviations: PVTT Portal vein tumor thrombus, HBsAg Hepatitis B surface antigen, PS Performance status, ECOG Eastern Cooperative Oncology Group, AFP Alpha-fetoprotein, PLT Platelet, HGB Hemoglobin, TBIL Total bilirubin, ALB Albumin, ALT Alanine aminotransferase, AST Aspartate aminotransferase 


\section{Characteristics}

$\geq 50$

$<50$

Abbreviations: PVTT Portal vein tumor thrombus, HBsAg Hepatitis B surface antigen, PS Performance status, ECOG Eastern Cooperative Oncology Group, AFP Alpha-fetoprotein, PLT Platelet, HGB Hemoglobin, TBIL Total bilirubin, ALB Albumin, ALT Alanine aminotransferase, AST Aspartate aminotransferase

Table 2

Significant covariates with respect to the survival and related log-rank test $P$-Values

\begin{tabular}{lll} 
Covariate & HR $(95 \%$ Cl for HR $)$ & $P$-value \\
\hline Age & $1.04(1.011-1.07)$ & 0.006796 \\
\hline Gender & $1.728(0.8304-3.598)$ & 0.1435 \\
\hline Stage T & $0.7244(0.2225-2.358)$ & 0.5924 \\
\hline Stage N & $0.943(0.5012-1.773)$ & 0.8546 \\
\hline Stage M & $0.936(0.435-2.014)$ & 0.8656 \\
\hline Types of PVTT & $0.518(0.276-0.971)$ & 0.0403 \\
\hline HBsAg & $0.989(0.9302-1.052)$ & 0.7339 \\
\hline Child-Pugh classification & $1.914(1.036-3.537)$ & 0.0243 \\
\hline ECOG & $2.342(1.232-4.453)$ & 0.009441 \\
\hline AFP & $1(1-1)$ & 0.06224 \\
\hline PLT & $1(0.9963-1.005)$ & 0.8193 \\
\hline HGB & $0.9783(0.9628-0.994)$ & 0.006954 \\
\hline TBIL & $1.007(0.9957-1.018)$ & 0.2332 \\
\hline ALB & $0.918(0.8543-0.9865)$ & 0.01982 \\
\hline ALT & $0.997(0.993-1.002)$ & 0.2326 \\
\hline AST & $0.999(0.9981-1.001)$ & 0.5197
\end{tabular}

Abbreviations: PVTT Portal vein tumor thrombus, HBsAg Hepatitis B surface antigen, PS Performance status, ECOG Eastern Cooperative Oncology Group, AFP Alpha-fetoprotein, PLT Platelet, HGB Hemoglobin, TBIL Total bilirubin, ALB Albumin, ALT Alanine aminotransferase, AST Aspartate aminotransferase

The coefficients of the selected radiomic features are shown in Fig. 2. Features 1-4 consisted of radiomic features and the Rad_score was calculated using the following formula: Rad_score $=$ feature $1 \times$ 
The cutoff Rad-score value was - 0.1 , which was used to classify patients into the high-risk group (Radscore $\geq-0.1$ ) and low-risk group (Rad-score <-0.1). The survival curves of both groups are shown in Fig. 3 .

The combination of the radiomic features, clinical characteristics nomogram, and calibration curves is presented in Fig. 4. The AUC of the ROC for the clinical characteristics was 0.761 (Cl: $0.641-0.881$ ), and the AUC of the ROC was 0.859 (Cl: $0.770-0.948)$ when the radiomic features were combined with the clinical characteristics (Fig. 5).

\section{Discussion}

The application of radiomics has been extensively studied in esophageal cancer [24], non-small cell lung cancer [25], breast cancer [26], nasopharyngeal carcinoma [27], and rectal cancer [28], which indicates the potential of radiomics for predicting the efficacy of treatment or patient prognosis. Radiotherapyorientated CT imaging must be acquired prior to SBRT treatment of HCC with PVTT. Image data analysis of the pre-SBRT CT image is used to predict the OS of HCC with PVTT, to limit examinations and provide guidance for clinical treatment decisions. This knowledge provided the basis for this retrospective study. We developed and validated a nomogram based on a combination of radiomic features and clinical characteristics from localized CT performed prior to SBRT treatment to make individualized OS predictions in patients with HCC with PVTT. The nomogram included seven radiological features and five clinical features. The methodology implemented in this study is simple and reproducible because the features were generated from a validated package, which is freely available from the 3D slicer [23].

LASSO regression is suitable for the accurate analysis of large radiological features with relatively small sample sizes and its design can prevent overfitting of the model [29-30]. The regression coefficients of most features are reduced to zero during the model fitting process, making it easier to interpret the model, which allows for the identification of features closely related to OS. The radiological features identified successfully classified patients into high-risk and low-risk groups, based on the Rad_scores.

SBRT focusses on treating stage IIIA and IVB HCC with PVTT, which has a relatively short OS. The accurate prediction of the OS of patients with HCC with PVTT undergoing SBRT will typically benefit those with shorter OS periods the most. We aim to develop a new model in a future study, which will include patients with low-stage HCC treated with SBRT. TNM staging was not selected as a clinical feature related to OS in this study, because patients with HCC with PVTT belong to the late clinical stage, which makes it difficult to predict OS using clinical staging, since all patients have similar staging information. Alpha-fetoprotein was also not selected as a clinical feature to determine OS in this study, perhaps for the same reason. This probably contributed to the poor predictive value of the clinical parameters in this study. The combination of the radiomic features and clinical characteristics resulted in better performance for the estimation of OS [AUC $=0.859$ (Cl: 0.770-0.948)] than that with the clinical characteristics alone $[A U C=0.761(\mathrm{Cl}: 0.641-0.881)]$. The radiomic features effectively compensated for 
the deficiencies in the clinical characteristics. It also supported the value of radiomic features for the individual association between the OS of HCC with PVTT treated by SBRT.

The limitations of the study are that genomic characteristics were not considered. In recent years, genetic markers have been used to predict OS in patients with liver cancer in research settings [31].

Radiogenomics is a discipline that studies the relationship between image phenotypes and genomics. It has gradually emerged in the field of cancer research and continues to receive more attention [32-34]. Further research is necessary with a larger study population, to identify the associated genetic characteristics and predict the OS of patients more accurately. Another limitation of this feasibility study is the lack of validation based on independent data sets. The limited size of the survey cohort hindered the possibility of dividing it into training and testing groups, and a multi-center separate validation study is being planned to overcome these limitations.

\section{Conclusions}

This study demonstrated that the use of a nomogram combining radiologic features with clinical risk factors can individualize OS prediction in patients with HCC with PVTT who underwent SBRT.

\section{Abbreviations}

AUC: area under the curve; HCC: hepatocellular carcinoma; LASSO: least absolute shrinkage and selection operator; OS: overall survival; PVTT: portal vein tumor thrombosis; ROC: receiver operating characteristic; SBRT: stereotactic body radiotherapy.

\section{Declarations}

\section{Acknowledgements}

The authors thank Professor Jie Qiu and Fuanquan Zhang from Department of Radiation Oncology, Peking Union Medical College Hospital for editorial assistance.

\section{Funding}

No funding was received.

\section{Availability of data and materials}

The datasets used and analyzed during the current study are available from the corresponding author on reasonable request.

\section{Authors' contributions}


Guarantors of integrity of entire study, HP; study concepts/study design or data acquisition or data analysis/interpretation, all authors; manuscript drafting or manuscript revision for important intellectual content, all authors; approval of final version of submitted manuscript, all authors; agrees to ensure any questions related to the work are appropriately resolved, all authors; literature research, KW, HP; clinical studies, all authors; statistical analysis, KW, YJS., HP.; and manuscript editing, KW, YJS, HP.

\section{Ethics approval and consent to participate}

This study was approved by the Ethics Committee of SAHZU, and was carried out in accordance with the Declaration of Helsinki.

\section{Consent for publication}

Not applicable.

\section{Competing interests}

The authors declare that they have no competing interests.

\section{References}

1. Siegel RL, Miller KD, Jemal A. Cancer Statistics. 2017. CA Cancer J Clin. 2017; 67(1):7-30.

2. Chen W, Zheng R, Baade PD, et al. Cancer statistics in China, 2015. CA Cancer J Clin. 2016;66(2):115-32.

3. Costentin CE, Ferrone CR, Arellano RS, et al. Hepatocellular Carcinoma with Macrovascular Invasion: Defining the Optimal Treatment Strategy. Liver Cancer. 2017;6(4):360-74.

4. Yuan BH, Yuan WP, Li RH, et al. Propensity score-based comparison of hepatic resection and transarterial chemoembolization for patients with advanced hepatocellular carcinoma. Tumour Biol. 2016;37(2):2435-41.

5. Zhang ZM, Lai EC, Zhang C, et al. The strategies for treating primary hepatocellular carcinoma with portal vein tumor thrombus. Int J Surg. 2015;20:8-16.

6. Chan SL, Chong CC, Chan AW, Poon DM, Chok KS. Management of hepatocellular carcinoma with portal vein tumor thrombosis: Review and update at 2016. World J Gastroenterol. 2016;22(32):7289300 .

7. Zhong JH, Peng NF, You XM, et al. Tumor stage and primary treatment of hepatocellular carcinoma at a large tertiary hospital in China: A real-world study. Oncotarget. 2017;8(11):18296-302.

8. Schöniger-Hekele M, Müller C, Kutilek M, et al. Hepatocellular carcinoma in Central Europe: prognostic features and survival. Gut. 2001;48(1):103-9.

9. Cabibbo G, Enea M, Attanasio $M$, et al. A meta-analysis of survival rates of untreated patients in randomized clinical trials of hepatocellular carcinoma. Hepatology. 2010;51(4):1274-83. 
10. Koo JE, Kim JH, Lim YS, et al. Combination of transarterial chemoembolization and threedimensional conformal radiotherapy for hepatocellular carcinoma with inferior vena cava tumor thrombus. Int J Radiat Oncol Biol Phys. 2010;78(1):180-7.

11. Fujino $H$, Kimura $T$, Aikata $H$, et al. Role of 3-D conformal radiotherapy for major portal vein tumor thrombosis combined with hepatic arterial infusion chemotherapy for advanced hepatocellular carcinoma. Hepatol Res. 2015;45(6):607-17.

12. Kubo K, Kimura $\mathrm{T}$, Aikata $\mathrm{H}$, et al. Long-term outcome of stereotactic body radiotherapy for patients with small hepatocellular carcinoma: Long-term outcome of SBRT for HCC. Hepatol Res. 2018;48(9):701-7.

13. Kellock T, Liang T, Harris A, et al. Stereotactic body radiation therapy (SBRT) for hepatocellular carcinoma: imaging evaluation post treatment. Br J Radiol. 2018;91(1085):20170118.

14. Miften M, Vinogradskiy Y, Moiseenko V, et al. Radiation Dose-Volume Effects for Liver SBRT. Int J Radiat Oncol Biol Phys. 2018;119:45-5.

15. Kubo K, Kimura T, Aikata $\mathrm{H}$, et al. Long-term outcome of stereotactic body radiotherapy for patients with small hepatocellular carcinoma: Long-term outcome of SBRT for HCC. Hepatol Res. 2018;48(9):701-7.

16. Larue R, Defraene G, De Ruysscher D, et al. Quantitative radiomics studies for tissue characterization: a review of technology and methodological procedures. Br J Radiol. 2017;90(1070):20160665.

17. Cook GJR, Azad G, Owczarczyk K, et al. Challenges and Promises of PET Radiomics. Int J Radiat Oncol Biol Phys. 2018;102(4):1083-9.

18. Parmar C, Grossmann P, Rietveld D, et al. Radiomic machine learning classifiers for prognostic biomarkers of head and neck cancer. Front Oncol. 2015;3(5):272.

19. Limkin E, Sun R, Dercle $L$, et al. Promised and challenges for the implementation of computational medical imaging (radiomics) in oncology. Ann Oncol. 2017;28(6):1191-2006.

20. Scalco E, Rizzo G. Texture analysis of medical images for radiotherapy applications. Br J Radiol. 2017;90(1070):20160642.

21. Sanduleanu S, Woodruff HC, De Jong EEC, et al. Tracking tumor biology with radiomics: A systematic review utilizing a radiomics quality score. Radiother Oncol. 2018;127(3):349-60.

22. Bruix J, Sherman M. Management of hepatocellular carcinoma. Hepatology. 2005;42(5):1208-36.

23. Fedorov A, Beichel R, Kalpathy-Cramer J, et al. 3D Slicer as an image computing platform for the Quantitative Imaging Network. Magn Reson Imaging. 2012;30(9):1323-41.

24. Antunovic L, De Sanctis $R$, Cozzi $L$, et al. PET/CT radiomics in breast cancer: promising tool for prediction of pathological response to neoadjuvant chemotherapy. Eur J Nucl Med Mol Imag. 2019;46(7):1468-77.

25. Peng H, Dong D, Fang MJ, et al. Prognostic Value of Deep Learning PET/CT-Based Radiomics: Potential Role for Future Individual Induction Chemotherapy in Advanced Nasopharyngeal Carcinoma. Clin Cancer Res. 2019;25(14):4271-9. 
26. Giannini V, Mazzetti S, Bertotto I, et al. Predicting locally advanced rectal cancer response to neoadjuvant therapy with (18)F-FDG PET and MRI radiomics features. Eur J Nucl Med Mol Imag. 2019;46(4):878-88.

27. Cook GJ, Yip C, Siddique M, et al. Are pretreatment 18F-FDG PET tumor textural features in non-small cell lung cancer associated with response and survival after chemoradiotherapy? J Nucl Med. 2013;54(1):19-26.

28. Pyka T, Bundschuh RA, Andratschke N, et al. Textural features in pre-treatment [F18]-FDG-PET/CT are correlated with risk of local recurrence and disease-specific survival in early stage NSCLC patients receiving primary stereotactic radiation therapy. Radiat Oncol. 2015;10:100.

29. Tibshirani R. The lasso method for variable selection in the Cox model. Stat Med. 1997;16(4):38595.

30. Gui J, Li H. Penalized Cox regression analysis in the high-dimensional and low-sample size settings, with applications to microarray gene expression data. Bioinformatics. 2005;21(13):3001-8.

31. Golobschwarzl N, Krassnig S, Toeglhofer AM, et al. New liver cancer biomarkers: PI3K/AKT/mTOR pathway members and eukaryotic translation initiation factors. Eur J Cancer. 2018;56(5):56-70.

32. Hong EK, Choi SH, Shin DJ, et al. Radiogenomics correlation between MR imaging features and major genetic profiles in glioblastoma. Eur Radiol. 2018;28(10):1-12.

33. Rattay T, Symonds RP, Shokuhi S, et al. The Patient Perspective on Radiogenomics Testing for Breast Radiation Toxicity. Clin Oncol. 2018;30(3):151-7.

34. Rutman AM, Kuo MD. Radiogenomics. Creating a link between molecular diagnostics and diagnostic imaging. Eur J Radiol. 2009;70(2):0-241.

\section{Figures}




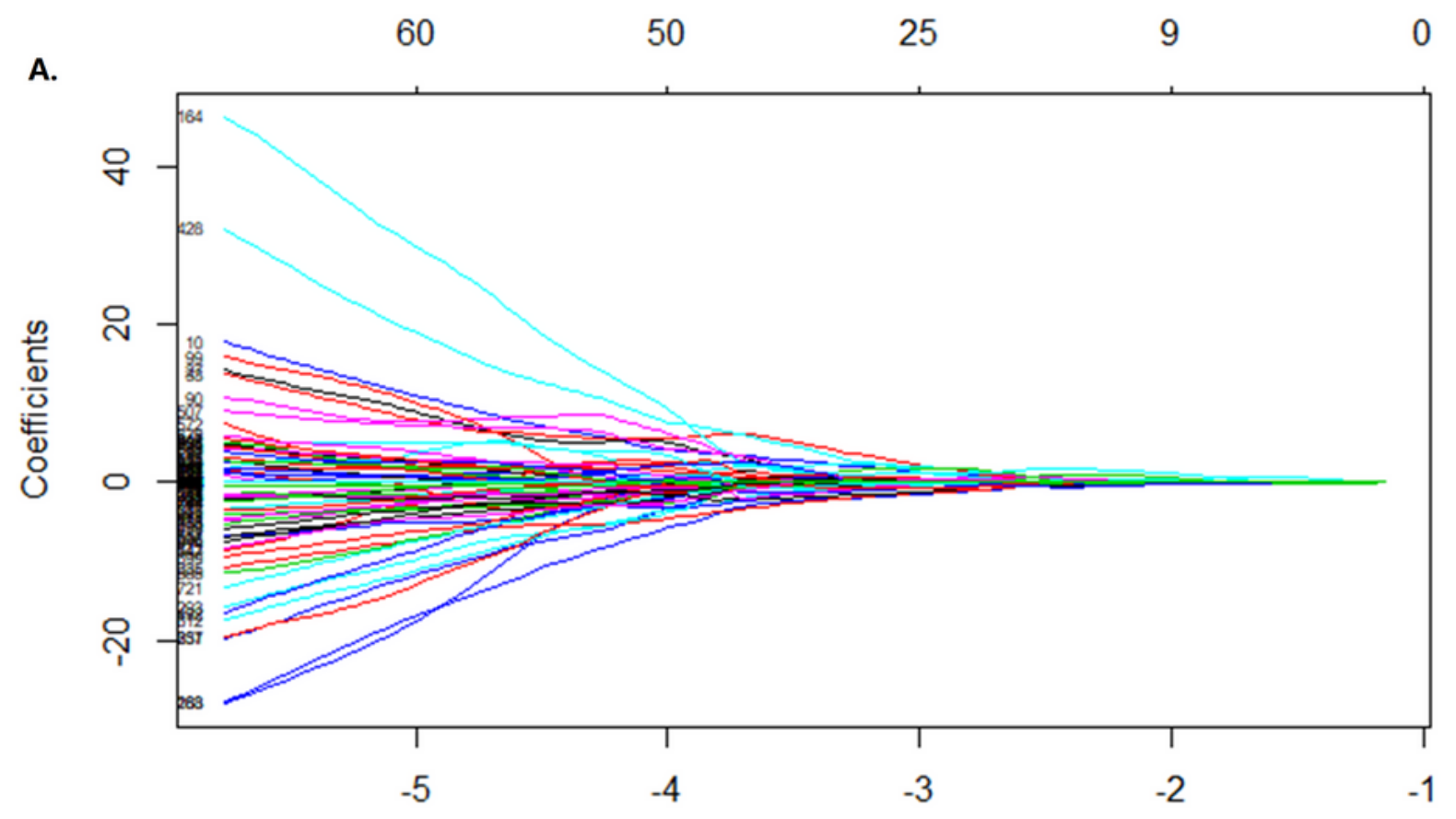

Log Lambda
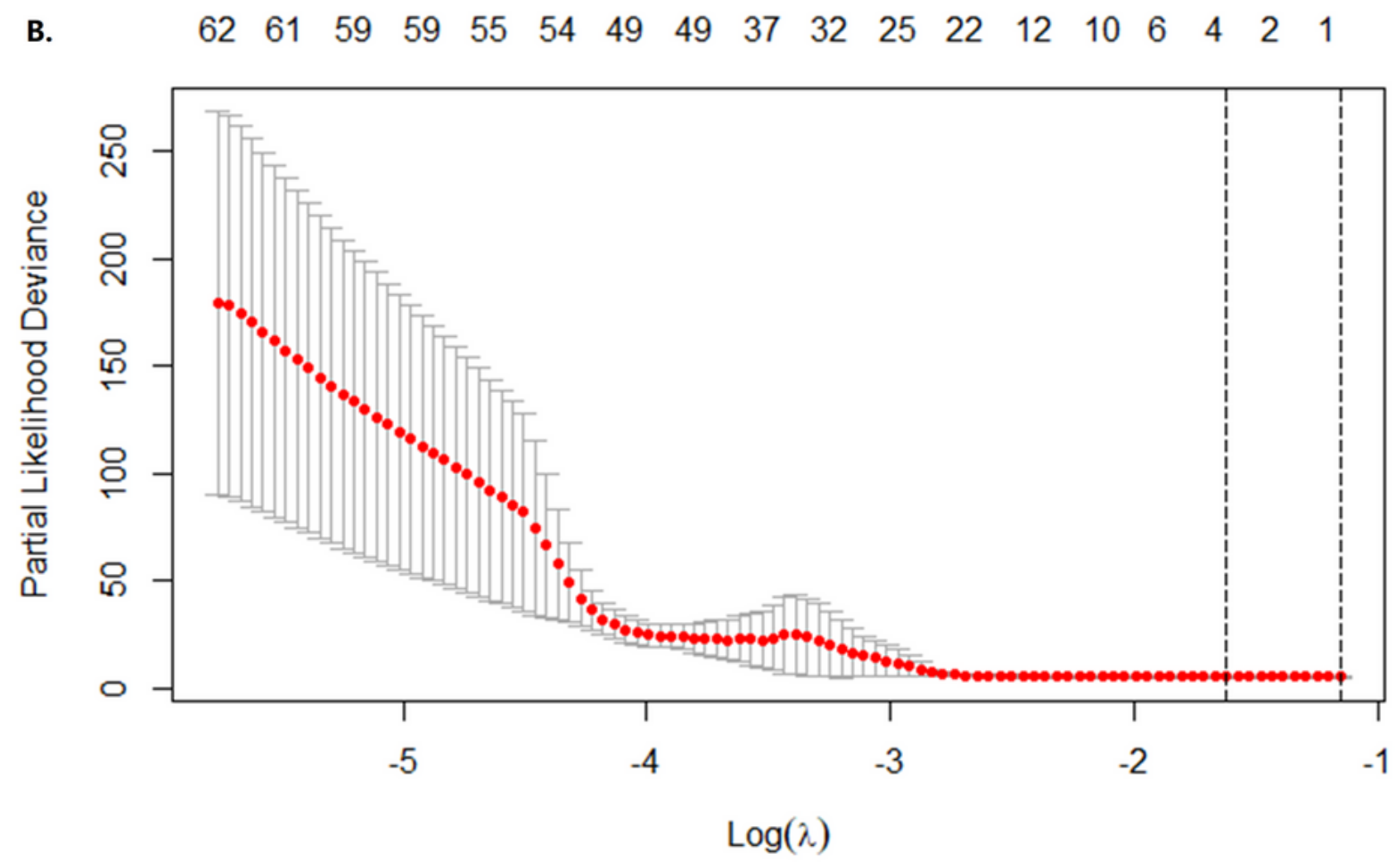

\section{Figure 1}

LASSO coefficient profiles of the 851 texture features (A) Tuning parameter $(\lambda)$ selection in the LASSO model used 10-fold cross-validation with minimum criteria (B). LASSO: least absolute shrinkage and selection operator 


\section{feature 4}

feature 3

feature2

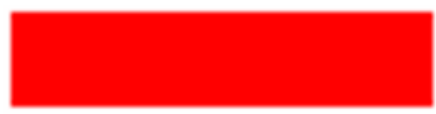

feature 1

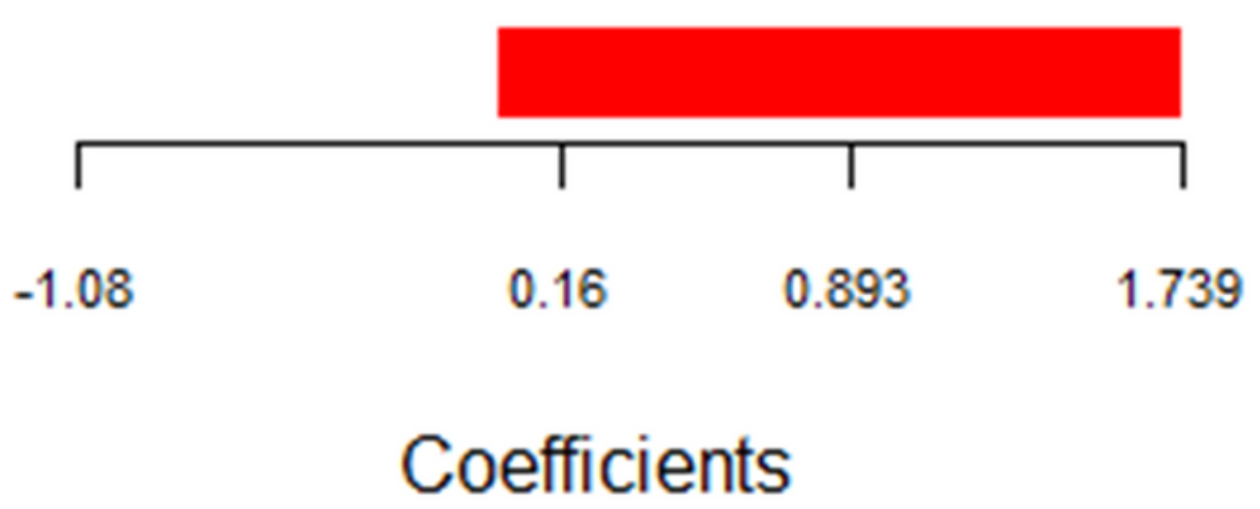

Figure 2

Histograms showing the role of individual features contributing to the radiomic features. Contributing features are plotted on the $y$-axis, and their LASSO analysis coefficients are plotted on the x-axis. LASSO: least absolute shrinkage and selection operator 


\section{Strata + group=high-risk $++\cdot$ group=low-risk}
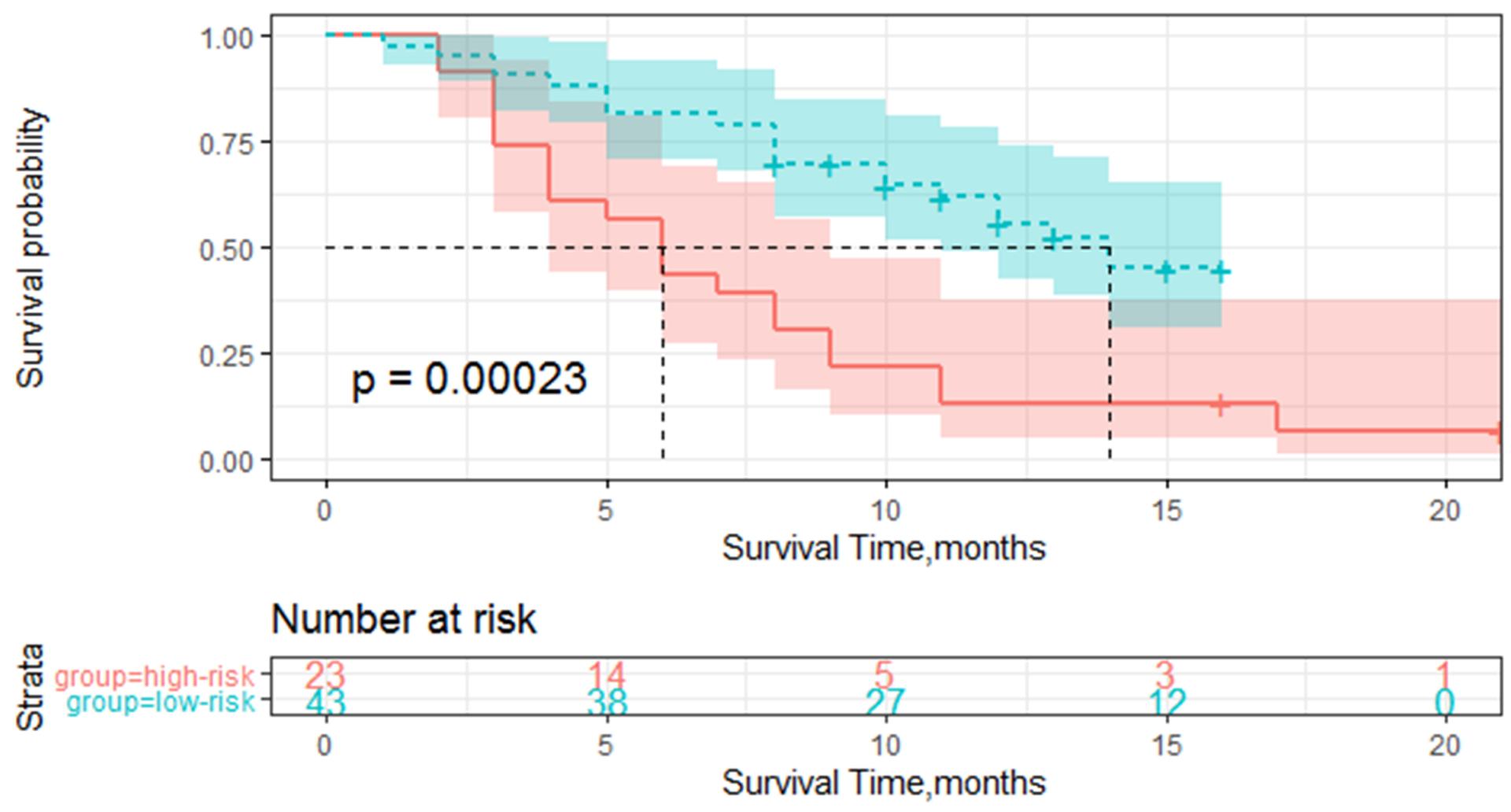

Figure 3

Survival curve of the high and low-risk groups based on the radiomics score classification. 
A.

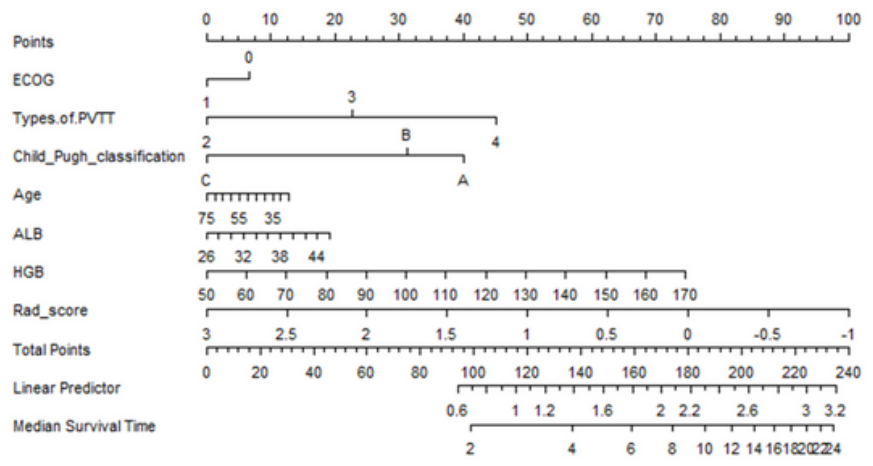

B.

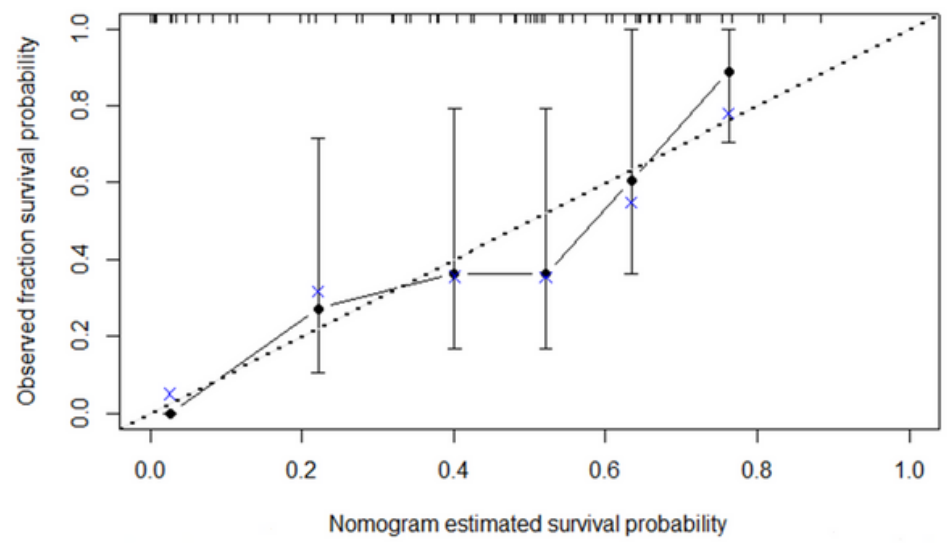

c.

Points

ECOG

Types.ot.PVTT

Child_Pugh_classification

Age

ALB

HGB

Total Points

Linear Predictor

Median Survival Time
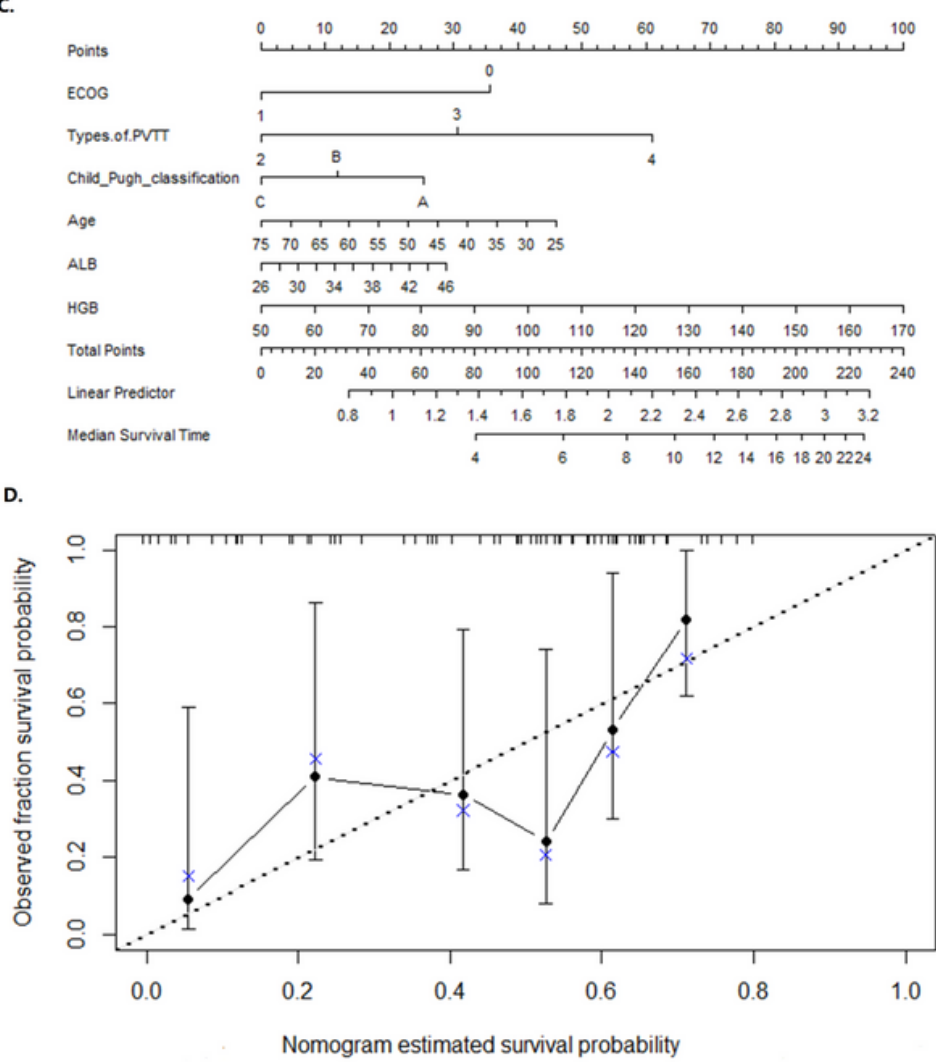

\section{Figure 4}

The nomogram of the combination of the radiomic features and clinical characteristics $(A)$ and the associated calibration curve for the combination of the radiomic features and clinical characteristics (B) The nomogram of the clinical characteristics (C) and the associated calibration curve for clinical characteristics (D). 

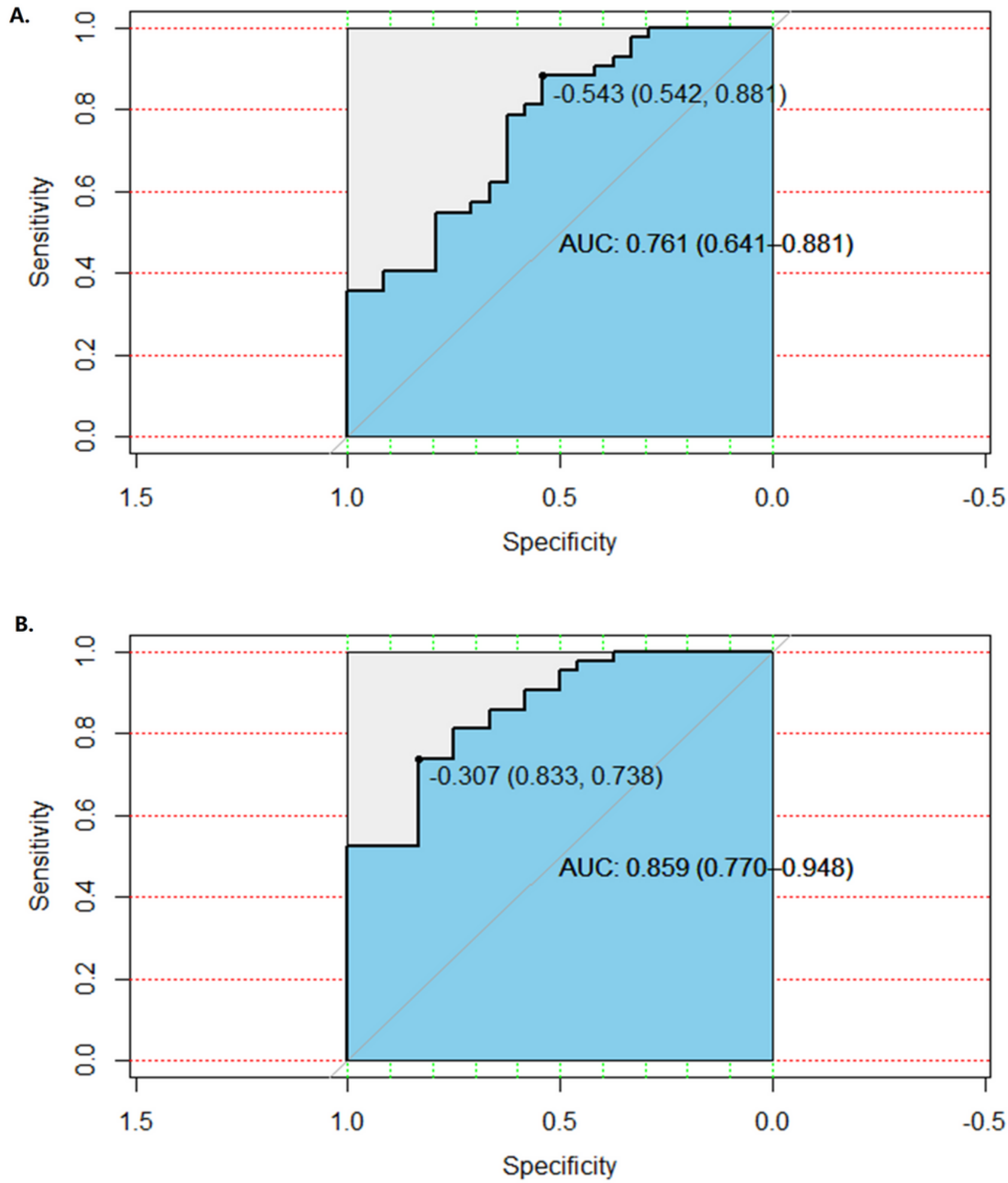

\section{Figure 5}

The AUC curve of clinical characteristics (A) and the combined radiomic features and clinical characteristics (B). AUC: area under the curve. 